\title{
Handwritten Chinese Character Recognition Technology based on CMAC Neural Network
}

\author{
Yan Shen, Lina Liu, Guoqiang Li \\ Department of Applied Mathematics, Collage of science, Harbin Engineering University, Harbin, 150001, China \\ shenyan@hrbeu.edu.cn, liulina@hrbeu.edu.cn, Liguoqiang84@hrbeu.edu.cn
}

\begin{abstract}
A character recognition technology based on CMAC (Cerebellar Model Articulation Controller) neural network was proposed, considering the neural network with the characteristics of stability and high distinctiveness. The handwriting recognition system interface was designed and developed, which provide a tools for theoretical application. Simulation results showed that the character recognition technology based on CMAC neural network, compared with that based on BP neural network, was accurate in the character recognition rate and character extraction. Besides, the method in the paper can be applied to any similar sign recognition.
\end{abstract}

Keywords - CMAC neural network; Character feature extraction; Direction feature; Spherical feature

\section{INTRODUCTION}

Character recognition ${ }^{[1][2]}$ as a means of information processing has broad application background and the huge market demand, which has great significance in a letter sorting, authentication, archaeological heritage and other aspects. Since the 80's, the studying of neural networks has a very rapid progress. Neural network has strong ability of learning knowledge and classification and has a high faulttolerance and robustness, complex decision-making region in the arbitrary feature space can be formed, it has the selforganization and self-learning function, so the constraints of the traditional pattern recognition were greatly broadened, these characteristics have contributed to the character pattern recognition. At present, there is not the precedent about CMAC neural network ${ }^{[3][4]}$ applications in the character recognition, and BP neural network ${ }^{[5]}$ is more commonly used. So, CMAC neural network model was compared with the traditional BP neural network model, whose results show that CMAC neural network can effectively identify handwritten character after the character feature was extracted.

\section{CMAC NEURAL NETWORK}

According to the biological model of the cerebellum, CMAC was introduced by J.S.Albus in 1975, which was successfully applied in control domain and signal processing. It took the input space which contains a large number of external stimulation pattern mapped to a storage space which contains little reflection pattern, and the similar vectors in the input space are mapped to the storage space are similar, so CMAC has a better integrated and generalization capacity. In addition, its training algorithm is simple and convergence speed is much faster than BP neural network.

Simply to say, CMAC can be described as a computing device, as shown in figure 1 . When the input vector is $S$, the output vector is $Y=F(S)$, CMAC approximated nonlinear function $y=f(s)$ by using two primary mappings:

$$
\left\{\begin{array}{l}
\rho(S) \Rightarrow A \\
F(A) \Rightarrow Y
\end{array}\right.
$$

A

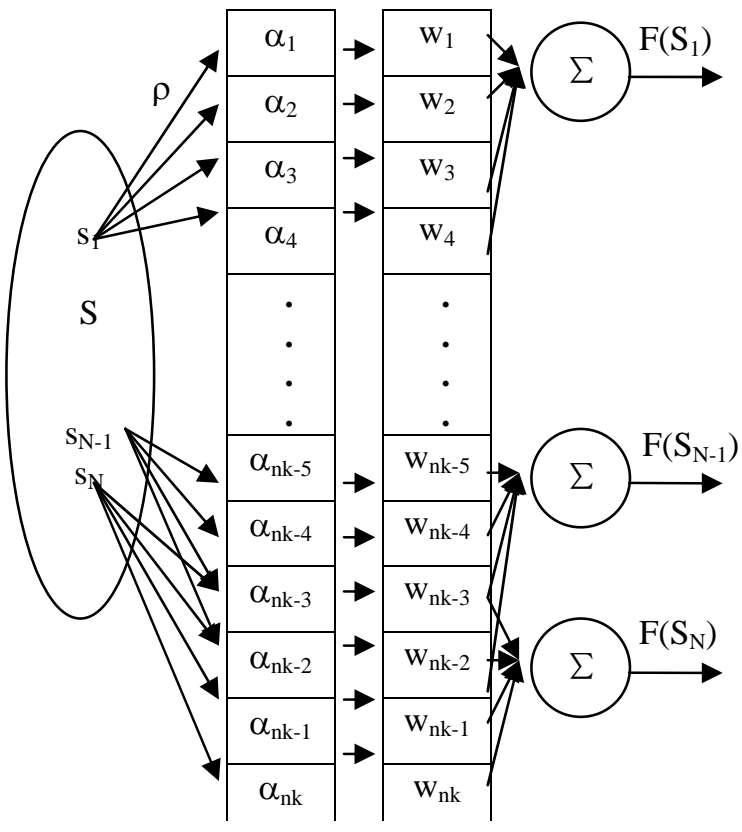

Fig.1 The function structure of CMAC neural network

Where $S$ is a continuous $\mathrm{N}$-dimensional input space, $Y$ is $\mathrm{M}$-dimensional output space. $A$ is an $\mathrm{N}$-dimensional association store vector-space. In fact, $A$ is a large storage address form, When $S$ is an input vector, the mapping function $\rho$ will point to a few (such as $C$ ) memory address in form $A$, and these addresses express that inspired association units in $A$. For any given input, the number of inspired units $(C)$ is a fixed parameter designed in CMAC, It can be seen that if a large association storage space $A$ is adopted on the input pattern, the greater $C$ in the storage 
vector space, more obvious enhancement of association capability. Let $\alpha$ be association storage vector in $A$, for a certain input, the number of non-zero element is $C$.Figure 1 depicts the function structure of CMAC neural network $(C=4)$. Usually,

$$
\alpha=A(s)
$$

For the standard CMAC networks, $\alpha$ only contains the binary element. The gain output $Y$ is calculated by association storage vector $A(s)$ which can map into an adjustable weight vector, so

$$
Y=F(A)=F\left(\alpha^{T} w\right)=\sum_{i=1}^{N} w_{i} F_{i}(A(s))
$$

LMS algorithm ${ }^{[6]}$ is usually used to train CMAC network.

\section{CHARACTER RECOGNITION}

The process of handwritten character recognition: At first, handwritten board input device is used, such as collection of characters to write the coordinates of sequence, and pre-treatment coordinates collected in order to improve the accuracy of feature extraction. Then the features of handwritten character with stability and high distinction degree are analyzed, and extraction algorithm is designed. Study of the characters, the establishment of character template library, the introduction of effective classification algorithm to identify samples to be compared with the template, and ultimately determine the types of characters, the characters are not identified for the study sample as a template into the database, a closed-loop feedback recognition system is formed.

\section{A. Coordinate collection}

When characters were written, the trajectory of nib was recorded on the handwritten board. The mobile trajectory of nib was generally described by its $x, y$ coordinates and the rise and fall state of pen on the board, the rise state of the pen divided mobile trajectories of nib into a number of strokes, that is, the trajectories was formed from fall state of pen to rise state of pen. As a result, the set of strokes was use to express handwritten character, each point sequence was used to express the stroke. The number of strokes depended on stroke length and sampling frequency, the value of each point coordinates $(x, y)$ in the handwritten board was decided by the location of nib and the resolving power of handwritten board.

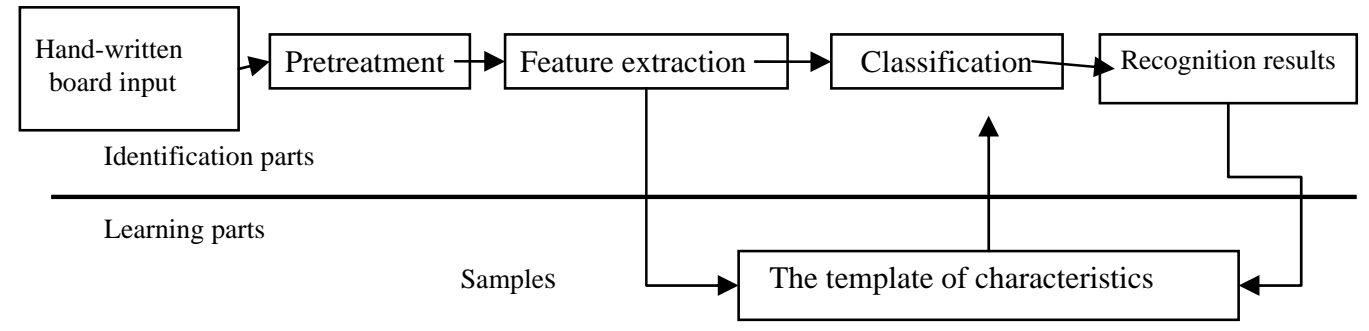

Fig.2 Flowchart of character recognition based on neural network

\section{B. The standardization of character image}

The outline of digital image was calculated by the method of nearest neighborhood ${ }^{[7]}$. The refining of the character strokes was used in many of the pretreatment process of character recognition, which divided character into the thread of single pixel. This will lead to distortion of character, In addition, the refining of the character need several operations and affect the processing speed. This paper only applied in the outline of character, not the refining of character, because the outline of character includes not only the characteristics of the strokes, but also the relative stability.

The gravity center of character is moved to the center of the grid. Let $f(i, j)$ denote dot-matrix of input character, $\mathrm{u} \rightarrow \mathrm{d}$ denotes height, $1 \rightarrow r$ denotes width, then the gravity center coordinates of character is as follows:

$$
G_{i}=\frac{\sum_{j=u}^{d} \sum_{i=1}^{r} i f(i, j)}{\sum_{j=u}^{d} \sum_{i=1}^{r} f(i, j)} \quad G_{j}=\frac{\sum_{j=u}^{d} \sum_{i=1}^{r} j f(i, j)}{\sum_{j=u}^{d} \sum_{i=1}^{r} f(i, j)}
$$

The center of character is moved to the center of $16 \times 16$ dot-matrix, and character is mapped to the $16 \times 16$ dot-matrix space using transform of amplification or demagnification.

For the character images $f(i, j)$, the point $(i, j)$ is the point of character outline, the points in the four-neighborhood of the black pixel point (i,j) was scanned, as long as one point in the four-neighborhood is not black, which set gray-value is 1 that expresses black. Gray-value of the other point $(\mathrm{i}, \mathrm{j})$ is 0 that expresses white. The complexity of this method was decreased, the style of writing and the shape of character were maintained, were not distorted, so the characteristics are also relatively stable. The size of digital images was taken the $16 \times 16$ dot-matrix after standardization (started from upper left corner). Figure 3, figure 4 respectively shows the character image after 
standardization and the dot-matrix $(16 \times 16)$ of input

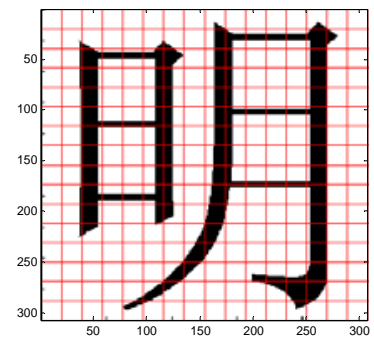

Fig.3 the character image after standardization

This paper makes a statistical analysis on 1000 Chinese song typeface characters which are standardized, the number of times which 1 appears in $16 \times 16$ dot-matrix is approximately between 0 96. 0 96 are equally divided into 8 groups and the histogram is made. The frequence of characters in two groups (36 48, 48 60) is relatively high as shown in figure 5 . This shows that characters in two groups (36 48, 48 60) are difficult identified, and the iterative times of CMAC neural network are correspondingly increased in the character recognition process. Characters in other groups are normally treated. The statistical condition of character is shown in figure 5.

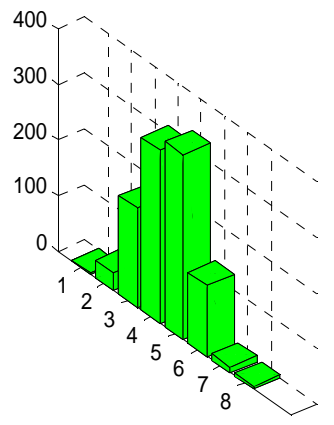

Fig.5 The statistical condition of character

\section{The feature extraction of handwritten character}

In this paper, the direction feature and spherical feature ${ }^{[8]}$ were used to extract handwritten character feature.

1) direction feature

Image matrix is transformed into a unified matrix of $16 \times 16$ by binaryzation and normalization. Whether printed or handwritten, characters are all strokes, and are the onedimensional structure on the two-dimensional plane. Local directed line segment can be used as feature, Kirsch template (Kirsch operator) that is a simple step-edge detection operator, which is used to test the feature. For each pixel $(i, j)$ of digital image, its gray-value change of 8neighbor points are inspected. If the maximum of all the differences between weighted sums of 3-neighbor points which are continuously shifted around the point $(i, j)$ and weighted sums of the remaining 5-neighbor points are bigger than a certain threshold, it will indicate that edge direction $^{[9]}$ exists, which can be expressed as: character.

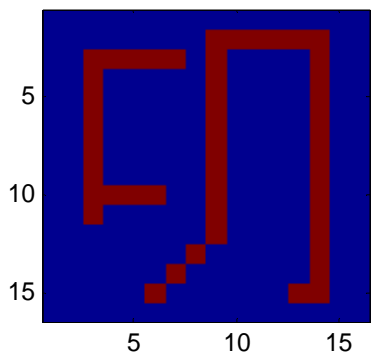

Fig.4 The dot-matrix $(16 \times 16)$ of input character

$$
K(i, j)=\max \left\{1, \max _{0 \leq k \leq 7}\left\{\left|5 S_{k}-3 T_{k}\right|\right\}\right\}
$$

Where $S_{k}=A_{k}+A_{k+1}+A_{k+2}, T_{k}=A_{k+3}+A_{k+4}+A_{k+5}+$ $A_{k+6}+A_{k+7}, A_{k}$ is the gray-value of point, and the subscript of $A$ is calculated by module 8 .

\begin{tabular}{|c|c|c|}
\hline$A_{0}$ & $A_{1}$ & $A_{2}$ \\
\hline$A_{7}$ & $(\mathrm{i}, \mathrm{j})$ & $A_{3}$ \\
\hline$A_{6}$ & $A_{5}$ & $A_{4}$ \\
\hline
\end{tabular}

Fig.6 The 8- neighborhood $A_{k}(k=0,1,2, \ldots, 7)$ of pixel point (i,j)

\begin{tabular}{|c|c|c|c|c|c|c|}
\hline 5 & 5 & 5 & \multirow{3}{*}{$H$} & -3 & -3 & -3 \\
\hline-3 & 0 & -3 & & -3 & 0 & -3 \\
\hline-3 & -3 & -3 & & 5 & 5 & 5 \\
\hline & & & & & & \\
\hline-3 & 5 & 5 & \multirow{3}{*}{$R$} & -3 & -3 & -3 \\
\hline-3 & 0 & 5 & & 5 & 0 & -3 \\
\hline-3 & -3 & -3 & & 5 & 5 & -3 \\
\hline & & & \multirow{4}{*}{$V$} & & & \\
\hline-3 & -3 & 5 & & 5 & -3 & -3 \\
\hline-3 & 0 & 5 & & 5 & 0 & -3 \\
\hline-3 & -3 & 5 & & 5 & -3 & -3 \\
\hline & & & \multirow{4}{*}{$L$} & & & \\
\hline-3 & -3 & -3 & & 5 & 5 & -3 \\
\hline-3 & 0 & 5 & & 5 & 0 & -3 \\
\hline-3 & 5 & 5 & & -3 & -3 & -3 \\
\hline
\end{tabular}

Fig.7 The 8 templates of Kirsch operator

Four direction features are designed based on Kirsch template, includes that the horizontal feature $(\mathrm{H})$, vertical feature $(V)$, right diagonal feature $(R)$ and left diagonal feature (L). Calculation formula of four direction features is as follows:

$$
\begin{aligned}
& K(i, j)_{H}=\max \left\{\left|5 S_{0}-3 T_{0}\right|,\left|5 S_{4}-3 T_{4}\right|\right\} \\
& K(i, j)_{V}=\max \left\{\left|5 S_{2}-3 T_{2}\right|,\left|5 S_{6}-3 T_{6}\right|\right\} \\
& K(i, j)_{R}=\max \left\{\left|5 S_{1}-3 T_{1}\right|,\left|5 S_{5}-3 T_{5}\right|\right\} \\
& K(i, j)_{L}=\max \left\{\left|5 S_{3}-3 T_{3}\right|,\left|5 S_{7}-3 T_{7}\right|\right\}
\end{aligned}
$$

\section{2) Spherical feature}


In order to further improve dipartite degree of the character after calculating the direction feature of character, This paper made the character writing on the plane stretched and distorted, and then mapped it to the sphere, which achieved the reconstruction of space, made twodimensional matrix one-to-one mapping to the threedimensional matrix. It can also have a choice to highlight the details of certain characters and improve the character recognition rate of the specific character.

\section{Recognition model}

A certain number of song typeface Chinese characters are given after feature extraction, which are used as samples for training CMAC neural network, and the value of direction feature or spherical feature of each sample is as input, the category according to the character (the serial number of the character) is as the desired output. When the mean square error is smaller than threshold (0.001), training is terminated. For any character image, if its value of direction feature or spherical feature is extracted, it can be identified by trained CMAC neural network. The prediction model is

$$
y_{i}=\operatorname{net}_{i}(X) \quad i=1,2, \cdots 10
$$

Where net $_{i}()$ is non-linear function, can be CMAC neural network to fit, and the characters which are identified at $i-1$ times before and $i-1$ times are successively excluded in the training samples of generating net $_{i}(), i=2,3, \cdots, 10 . y_{i}$ is the serial number of the character, $X$ is the value of direction feature or spherical feature of input character.

\section{SYSTEM SIMULATION}

In order to verify the feasibility of the method, a system interface of Chinese character recognition was designed based on the identification theory of CMAC neural network. The main function of system interface is to identify handwritten Chinese character, which has some advantages such as identification of high precision, easy to use, etc. Figure 8 shows system interface of Chinese character recognition.

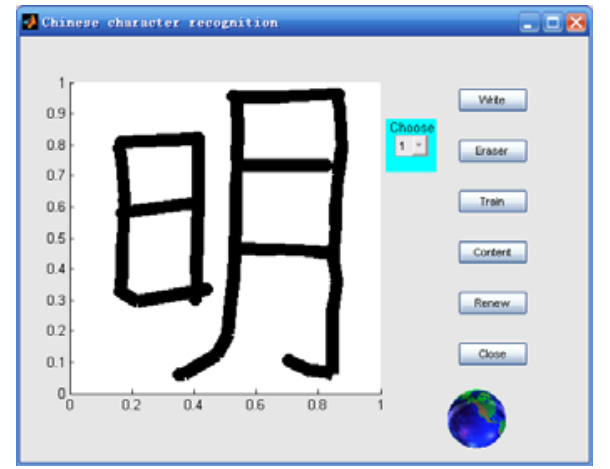

Fig. 8 The system interface of Chinese character recognition

At first, a image of handwritten Chinese character ('明’) input into system interface, and then the feature of the character is extracted, finally, the best matching top 10 characters are given by trained CMAC neural network which use 100 characters ( '辽','吉','黑','大','小','真','明','理',' 里','力','海','苏','儿','刀','道','倒','岛','石','楠','广','西','文','庆 ','川','贵','云','子','字','母','亲','客','网','事','藏','陕','甘','青',' 疆','镶','湘','雯','自','己','急','鸟','兽','级','击','老','用','张','长 ','扬','中','严','重','耳','笔','比','设','浙','安','福','江','东','南',' 湖','播','种','剑','数','天','师','花','甲','君','红','黄','蓝','绿','黑 ','总','夫','芳','因','龙','空','将','神','北','天','河','山','内','经',' 匡','络','员','是','雨') as a database. Figure 9 shows that Training situation of CMAC neural network.

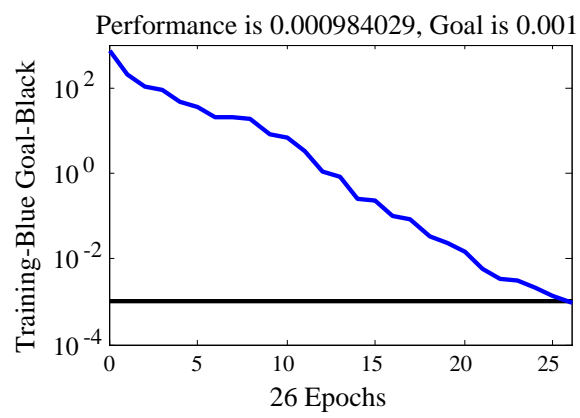

Fig.9 Training situation of CMAC neural network

Figure 10 and figure 11 respectively show that the edge direction of character and the direction feature of handwritten character “明” as follows:

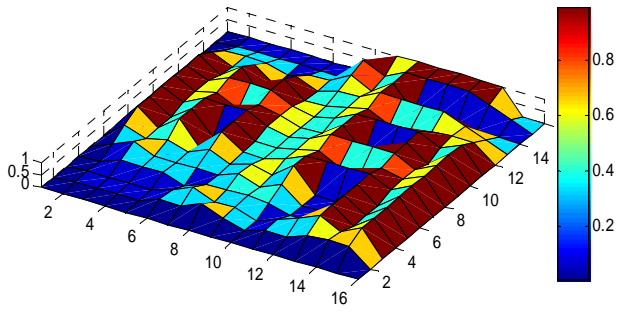

Fig.10 The edge direction of character
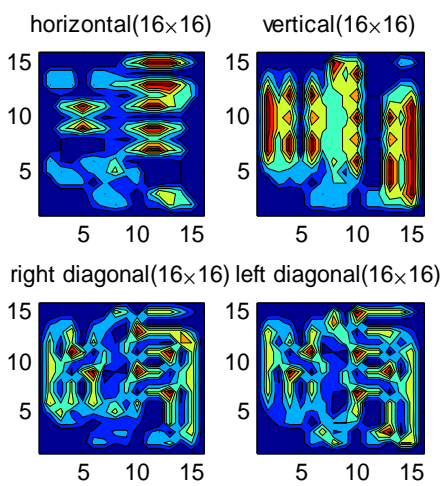

Fig.11 Direction feature

Figure 12 shows that the spherical feature of handwritten character and song typeface character. The left is a 
handwritten character “明”, and the right is the song typeface character “明”.

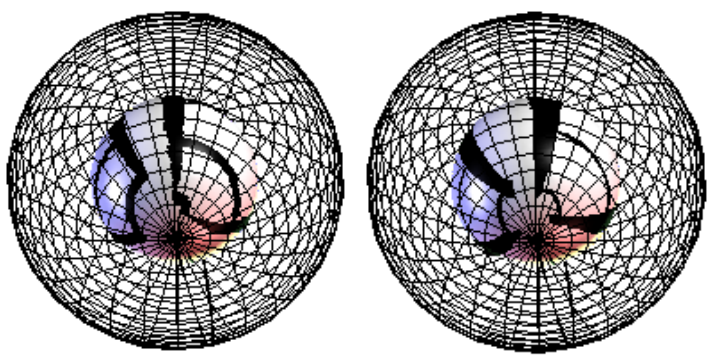

Fig.12 Spherical feature

The neural network theory is used to identify the character after character feature extraction. Figure 13 and Figure 14 respectively show the recognition results of threelayer BP neural network and CMAC neural network. it is not difficult to know that the effect of recognition of the CMAC neural network is better.

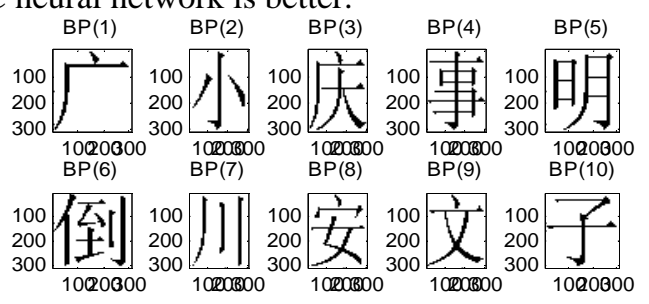

Fig.13 Character recognition results of BP neural network

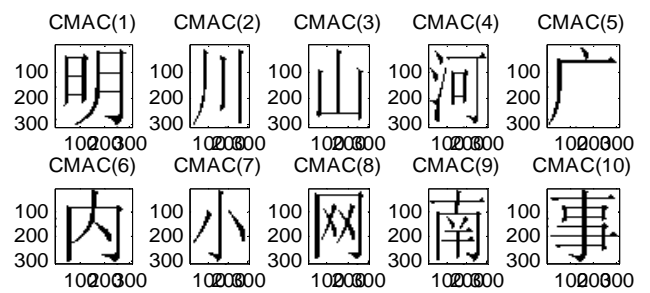

Fig.14 Character recognition results of CMAC neural network

The system recognizes 625 daily handwritten Chinese characters, whose training samples respectively based on 1000 song typeface characters and 2000 song typeface characters. As long as correct recognition result of input character appears in its best matching top 5 characters, the character can be identified. Table I shows the situation of character recognition as follows:

TABLE I. The results of experiment

\begin{tabular}{l|c|c}
\hline \multicolumn{1}{c|}{ Test text } & $\begin{array}{c}\text { Recognition rate } \\
(1000 \text { characters })\end{array}$ & $\begin{array}{c}\text { Recognition rat } \\
(2000 \\
\text { characters })\end{array}$ \\
\hline Test text1 (BP) & $83.68 \%$ & $68.64 \%$ \\
\hline $\begin{array}{l}\text { Test text2 } \\
\text { (CMAC) }\end{array}$ & $95.52 \%$ & $89.76 \%$ \\
\hline
\end{tabular}

\section{CONCLUSION}

2000 Chinese characters are used as a template for training in our study. It is found that the handwritten character recognition accuracy rate of writing neat, appropriate proportion and straight strokes was up to $89.76 \%$, however, the free writing recognition effect is not very good, to be corrected manually. With the development of the CMAC neural network theory and the depth study of the character feature extraction technology, CMAC neural network will be better used in the field of handwritten character recognition and has a very good application prospects.

\section{REFERENCES}

[1] Hong-Yuan Liao, Jun-Shon Huang and Shih-Ta Huang, "Twodimensional neural networks for handwritten Chinese character recognition”, International Joint Conference on Neural Networks, Vol.3, pp. 579 - 584, 7-11 Jun 1992.

[2] Hailong Liu and Xiaoqing Ding,"Handwritten Character Recognition Using Gradient Feature and Quadratic Classifier with Multiple Discrimination Schemes", Proceedings of the 2005 Eight International Conference on Document Analysis and Recognition, Vol. 1, pp. 19 - 23, 29 Aug-1 Sep 2005.

[3] J. S. Albus, "A new approach to manipulator control: The cerebellar model articulation controller (CMAC)", Journal of Dynamic Systems, Measurement and Control, Transactions of ASME, pp.220-227, Sep 1975.

[4] J. S. Albus, "Data storage in the cerebellar model articulation controller (CMAC)", Journal of Dynamic Systems, Measurement and Control, Transactions of ASME, pp.228-233, Sep 1975.

[5] A. Khawaja, S. Tingzhi, N.M. Memon and A. Rajpar, "Recognition of printed Chinese characters by using Neural Network", IEEE International Multitopic Conference, pp. 169 - 172, 23-24 Dec. 2006.

[6] B. Widrow, J. McCool and M.Bal1, "The complex LMS algorithm”, Proceedings of the IEEE, vol. 63, pp. 719-720, Apt. 1975.

[7] K. Gowda and G. Krishna, "The condensed nearest neighbor rule using the concept of mutual nearest neighborhood", IEEE Transactions on Information Theory, Vol.25, pp. 488 - 490, Jul 1979.

[8] Y.C.Shiu and Shaheen Ahmad, "3D Location of Circular and Spherical Features by Monocular Model-Based Vision”, IEEE International Conference on Systems, Man and Cybernetics, vol.2, pp. 576 - 581, 14-17 Nov. 1989.

[9] Chan Khue Hiang and S.S Erdogan, "A New Convolutional Map Feature Extraction for Character Recognition", International Joint Conference on Neural Networks, Vol. 4, pp. 2887 - 2892, 10-16 July 1999. 